\section{Esporotricose urbana: epidemia negligenciada no Rio de Janeiro, Brasil}

\author{
Urban sporotrichosis: a neglected epidemic in \\ Rio de Janeiro, Brazil
}

\author{
Margarete Bernardo Tavares da Silva 1 \\ Mônica Motta de Mattos Costa 1 \\ Carla Carrilho da Silva Torres 1 \\ Maria Clara Gutierrez Galhardo 1 \\ Antonio Carlos Francesconi do Valle ${ }^{1}$ \\ Mônica de Avelar F. M. Magalhães 2 \\ Paulo Chagastelles Sabroza ${ }^{3}$ \\ Rosely Magalhães de Oliveira ${ }^{3}$
}

\footnotetext{
${ }_{1}^{1}$ Instituto de Pesquisa Clínica Evandro Chagas, Fundação Oswaldo Cruz, Rio de Janeiro, Brasil.

2 Instituto de Comunicação

e Informação Científica e Tecnológica em Saúde, Fundação Oswaldo Cruz, Rio de Janeiro, Brasil. ${ }^{3}$ Escola Nacional de Saúde Pública Sergio Arouca, Fundação Oswaldo Cruz, Rio de Janeiro, Brasil.

Correspondência M. B. T. Silva Instituto de Pesquisa Clínica Evandro Chagas, Fundação Oswaldo Cruz.

Av. Brasil 4365, Rio de Janeiro, $R J$ 21040-900, Brasil. margarete.tavares@ipec. fiocruz.br
}

\begin{abstract}
In the scientific literature, sporotrichosis has traditionally been associated with agricultural work, since the causative agent is found naturally in the soil. However, cases have been reported recently in an urban area, related to zoonotic transmission. The current study aimed to contribute to knowledge on sporotrichosis in an urban area through an exploratory analysis of its socio-spatial distribution in Rio de Janeiro, Brazil, from 1997 to 2007, identifying the areas with the heaviest transmission. The database from the Health Surveillance Service at the Evandro Chagas Institute for Clinical Research, Oswaldo Cruz Foundation, was used to estimate incidence rates and spatial distribution. During the study period, 1,848 cases of sporotrichosis were reported, predominantly in adult women not currently in the labor market. The leading source of infection was wounds caused by domestic cats, which contributed to the spread of sporotrichosis in this urban area. Georeferencing of 1,681 cases showed a transmission belt along the border between the city of Rio de Janeiro and the adjacent municipalities in the Greater Metropolitan Area.
\end{abstract}

Sporotrichosis; Cat Diseases; Epidemiological Surveillance; Urbanization

\section{Introdução}

A esporotricose é uma micose subaguda ou crônica causada, na maior parte dos casos, por implantação traumática do fungo Sporothrix schenckii na pele 1 . A doença vem se tornando um problema de saúde pública no Estado do Rio de Janeiro, Brasil, em razão do aumento significativo de casos em seres humanos nos últimos anos 2,3,4,5,6,7,8,9,10,11.

A esporotricose pode acometer o ser humano de ambos os sexos, de qualquer faixa etária ou raça, independentemente de fatores individuais predisponentes ${ }^{12}$. Na literatura, a ocorrência da doença é predominantemente associada à ocupação profissional, afetando pessoas que lidam com a terra, particularmente em área rurais 1,13 . Porém, neste início de século, a ocorrência tem sido relacionada também à arranhadura e/ou mordedura de gatos, levando a surtos familiares, além de casos em profissionais que lidam com esses animais, como veterinários e auxiliares $7,14,15$.

No final do século XX, alguns autores afirmavam que havia poucos estudos epidemiológicos sobre a investigação epidemiológica da esporotricose 15,16,17. Alguns desses estudos abordavam os aspectos clínicos da doença em um determinado serviço de saúde 12,18, outros, de caráter epidemiológico, versavam especialmente sobre surtos localizados como o que ocorreu com mineradores na África do Sul 19. Bustamante \& Campos 20 
(p. 145) afirmam que "embora relatada há mais de um século, a esporotricose ainda permanece uma doença mal estudada". Schubach et al. 7 (p. 129) demonstram uma preocupação específica: "até agora não se sabe por que a esporotricose toma a proporção de uma zoonose emergente no Rio de Janeiro".

Este trabalho tem como objetivo descrever a ocorrência da epidemia de esporotricose humana em residentes de área urbana no Estado do Rio de Janeiro no período de 1997-2007, utilizando técnicas de análise exploratória de dados espaciais 21. Embora muitos estudos tenham sido desenvolvidos sobre o tema nesse estado 2,3,4,6,7,9,10, esta é a primeira vez em que uma abordagem espacial é utilizada para avaliar o problema.

\section{Materiais e métodos}

\section{Cenário do estudo}

O Estado do Rio de Janeiro ocupa uma área de $43.780,157 \mathrm{~km}^{2}$ e possui uma população de 15.989.929 habitantes (Instituto Brasileiro de Geografia e Estatística. Sinopse preliminar do censo demográfico 2010. http://www.ibge.gov. br/home/estatistica/populacao/censo2010/ sinopse, acessado em 10/Jul/2011), distribuída em 92 municípios, que são agrupados em oito regiões de governo. São elas: Região Metropolitana, Baixadas Litorâneas, Norte Fluminense, Noroeste Fluminense, Serrana, Centro-Sul Fluminense, Médio Paraíba e Costa Verde. Tal divisão está apoiada na Lei $n^{o}$. 1.227/1987, que aprovou o Plano de Desenvolvimento Econômico e Social 1988/1991 22. A Região Metropolitana é composta por 18 municípios; nela se encontra a segunda maior concentração urbana do país 23 .

Segundo o Plano Diretor de Regionalização, a Região Metropolitana 23 é subdividida em dois polos, em lados opostos da Baía de Guanabara: Região Metropolitana I, com 11 municípios (Rio de Janeiro, Belford Roxo, Duque de Caxias, Japeri, Magé, Mesquita, Nilópolis, Nova Iguaçu, Queimados, São João de Meriti e Seropédica) e Região Metropolitana II com sete municípios (Itaboraí, Maricá, Niterói, Rio Bonito, São Gonçalo, Silva Jardim e Tanguá).

As regiões Metropolitanas I e II concentram o maior número de habitantes, correspondendo em conjunto a 73,9\% da população total do estado. A Região Metropolitana I é a mais populosa $(61,7 \%)$, sendo o número de indivíduos que nela residem cinco vezes superior ao da Região Metropolitana II (12\%). Os municípios do Rio de Janeiro, Duque de Caxias, Niterói e Nova Iguaçu são os mais importantes centros urbanos do estado 23.
A população total do estado apresentou um crescimento significativo, passando de 12.807 .706 habitantes, em 1991, para 14.391.282 habitantes em 2000 e para 15.989.929 habitantes em 2010 23,24 (http://www.ibge.gov.br/home/estatistica/ populacao/censo2010/sinopse, acessado em 10/ $\mathrm{Jul} / 2011)$.

A distribuição da população é absolutamente desigual. Na Região Metropolitana I há uma concentração populacional representada por uma densidade demográfica (http://www.ibge. gov.br/home/estatistica/populacao/censo2010/ sinopse, acessado em 10/Jul/2011) de 2.848,44 habitantes $/ \mathrm{km}^{2}$, quase oito vezes superior à densidade demográfica média do estado, que é de 365,23 habitantes $/ \mathrm{km}^{2}$.

\section{Desenho do estudo}

Este estudo se caracteriza por ser uma análise exploratória sobre a distribuição de esporotricose humana diagnosticada em residentes no Estado do Rio de Janeiro, no período compreendido entre 1997 e 2007. O estudo transcorreu em duas etapas: na primeira etapa foi realizada uma análise descritiva dos casos de acordo com algumas características das pessoas acometidas; na segunda etapa, realizou-se uma análise espacial para evidenciar as áreas de concentração da doença.

Foram eleitos para este estudo todos os pacientes com diagnóstico de esporotricose no período de 1997-2007, residentes no Estado do Rio de Janeiro, tratados no Instituto de Pesquisa Clínica Evandro Chagas, Fundação Oswaldo Cruz (IPEC/Fiocruz). O diagnóstico foi pautado em informações clínicas, laboratoriais ou clínico-epidemiológicas. Entendeu-se por critério laboratorial de esporotricose o isolamento em cultivo do S. schenckii, com base em espécimes clínicas; por critério clínico-epidemiológico, as lesões compatíveis ${ }^{1}$ com história de trauma de matéria orgânica possivelmente contaminada ou contato com gato doente; e por critério clínico, lesões compatíveis com esporotricose e resposta clínica ao tratamento instituído.

As informações utilizadas foram oriundas da base de dados do Serviço de Vigilância em Saúde do IPEC/Fiocruz, gerada mediante análise de prontuários do atendimento ambulatorial do IPEC. Ao longo dos 11 anos analisados neste estudo, as variáveis que compunham tal base sofreram modificações 21 . Após a análise das variáveis existentes e a definição de quais entrariam no estudo, foi realizada uma pesquisa documental na totalidade de prontuários selecionados, com o objetivo de rever e completar todas as informações no banco de dados. 
Consideramos ser possível afirmar que a atual casuística de esporotricose humana registrada pelo IPEC corresponda a uma parcela expressiva do universo dos casos no Estado do Rio de Janeiro. Isso se deve ao conhecimento de que a esporotricose humana não é uma doença de notificação compulsória, e ao fato de o IPEC ser um serviço de referência nacional em micose, sendo o único local no estado onde são disponibilizados métodos diagnósticos, acompanhamento clínico e tratamento gratuito para a doença.

\section{Descrição segundo características} das pessoas

O perfil epidemiológico da esporotricose humana foi avaliado mediante análise de sua distribuição temporal e segundo variáveis: sexo, raça, faixa etária, ocupação, escolaridade, residência, fonte de infecção e presença de animal no ambiente domiciliar. Para esta finalidade, utilizouse o aplicativo estatístico SPSS 16.0 (SPSS Inc., Chicago, Estados Unidos).

Os registros referentes à variável ocupação foram agrupados segundo a situação das ocupações (IBGE. Classificação Nacional de Atividades Econômicas. http://www.cnae.ibge.gov, acessado em Ago/2011): (1) ocupações atuais bem definidas em atividade - com registros; (2) dona de casa; (3) estudante; (4) ignorado; (5) aposentado, pensionista e reformados; (6) atividades veterinárias; (7) autônomo; (8) desempregado; e (9) não se aplica - crianças fora da idade escolar. Para fins de análise, a variável original foi agregada em duas classes: fora do mercado de trabalho (2, 3, 5, 8 e 9) e formalmente empregado (1, 6 e 7$)$.

\section{Análise espacial}

Para análise da distribuição espacial, os casos foram georreferenciados por endereço de residência. O georreferenciamento 25,26 transcorreu em dois momentos. No primeiro momento, foi realizado para os residentes no Município do Rio de Janeiro, utilizando a ferramenta de Geocoding do programa ArcGis (http://www.esri.com/softwa re/arcgis/index.html). Para realizar tal procedimento, foi necessária a construção de um banco de dados com o campo endereço e uma malha de ruas adequada ao trabalho em ambiente de Sistema de Informação Geográfico (SIG).

Deve-se lembrar que em todo ambiente SIG 25 existe uma inexatidão na localização das coordenadas geográficas: o ponto atribuído ao caso não representa fielmente o endereço, mas está próximo. Dependendo da base de ruas, esse erro pode variar em alguns metros.
No primeiro momento, o programa georreferenciou automaticamente 55\% dos casos residentes na capital do estado. Para os endereços não encontrados, foi realizado o georreferenciamento semiautomático. O programa ofereceu algumas alternativas que foram aceitas pelo pesquisador. Ao final do procedimento, foram obtidos $72 \%$ dos casos georreferenciados no Município do Rio de Janeiro.

Posteriormente, foi realizado o georreferenciamento para os endereços não localizados na capital e para todos os demais residentes em outros municípios do estado. Para tanto, foi realizada uma busca individual dos endereços nos guias de ruas, listas telefônicas, mapas, sites de serviços gratuitos de localização, entre outros. Quando localizado, o endereço foi marcado como um ponto no programa gratuito Google Earth, permitindo assim a identificação das coordenadas de latitude e longitude. Foi utilizado o sistema de coordenadas projetadas UTM para América do Sul (SAD/69 - South American 1969 UTM Zone 23S).

As coordenadas de cada ponto identificadas no Google Earth foram inseridas num shapefile (shape), resultando em uma nova malha digital. O shapefile é um formato vetorial de dados geoespaciais que se utiliza em programas de SIG. Foi desenvolvido e regulamentado pela ESRI (Environmental Systems Research Institute. http:// webhelp.esri.com/arcgisdesktop/9.3/index. cfm?TopicName=Defining_a_shapefile\%27s_co ordinate_system, acessado em 17/Out/2011) com padrão aberto, o que permite interoperabilidade de dados entre vários programas de SIG.

Os casos foram agrupados em aquivos shape por agregados de ano de notificação em três períodos: 1997-2000, 2001-2004 e 2005-2007. A análise espacial foi realizada por meio da densidade de kernel 26,27, usando os programas ArcGis 9.0 e TerraView 3.2.0 (Instituto Nacional de Pesquisas Espaciais; http://www.dpi.inpe.br/terraview). Os parâmetros utilizados na analise de kernel foram os seguintes: casos agrupados por setor censitário, sendo definida uma grade sobre os eventos com 300 colunas e a função algoritma utilizada foi quártica, com um raio de mil metros, e 12 faixas de classificações de densidade dos casos. Posteriormente, essas faixas foram reagrupadas em três estratos de densidade e classificados em alta densidade (tons preto), média densidade (tom cinza-escuro) e baixa densidade (tom cinza-claro). A construção do mapa de kernel permitiu identificar as áreas com maior densidade de casos.

Para demarcação das áreas, foi utilizada a ferramenta vetorização no programa ArcGis 9.0. Transformando-se o arquivo em formato matri- 
cial do kernel em um arquivo formato vetorial, foi gerada uma nova shape, com polígonos demarcados pela borda externa de cada faixa de cor do kernel (Figura 1). Desse modo, permitiuse, com o uso da ferramenta de operações entre camadas, identificar os casos de esporotricose residentes em cada estrato de densidade previamente identificado.

Com os casos estratificados pela densidade de kernel, foram construídas análises de frequência para cada estrato de densidade (alta/média/ baixa) das seguintes variáveis: sexo, raça, faixa etária, situação no mercado de trabalho, escolaridade e fonte de infecção.

Com a sobreposição de camadas entre a malha de setores censitários e o polígono das faixas de kernel, foi possível identificar quais setores censitários tinham interseção com o limite do polígono estabelecido (Figura 1). Com tais setores identificados, foram realizadas análises sobre as características socioeconomicas, de infraestrutura e saneamento com dados do Censo Demográfico de 2000 do IBGE (http://www.ibge.gov.br).

O projeto 21 foi submetido ao Comitê de Ética em Pesquisa do IPEC/Fiocruz e aprovado em 7 de abril de 2009. O parecer consubstanciado 009/2009 tem registro no CONEP sob nº. 0001.0.009.031-09.

\section{Resultados}

Entre 1997 e 2007, foram diagnosticados e tratados 1.848 casos de esporotricose humana no Estado do Rio de Janeiro, dos quais 1.289 foram registrados no último quadriênio. A frequência observada para os últimos quatro anos representa o dobro de casos relativos aos sete anos anteriores (Figura 2).

Quanto às características sociodemográficas, os pacientes atendidos eram predominantemente mulheres $(66,9 \%)$, na faixa etária entre 21 e 60 anos (67,5\%). A razão entre mulheres e homens é de 2:1. A doença acometeu indivíduos de todas as "raças", predominando naqueles classificados como da raça branca. A distribuição da frequência de casos segundo anos de estudo indicou a ocorrência de casos da doença nos diferentes graus de escolaridade.

No que se refere às ocupações declaradas, excluímos nesta análise 94 registros categorizados como ignorados. Para essa variável, informações completas foram obtidas para 1.754 casos, dos quais $973(55,5 \%)$ estão formalmente no mercado de trabalho e $781(44,5 \%)$ estão fora. Incluemse nesta última categoria donas de casa, aposentados/pensionistas, desempregados, estudantes e crianças (Tabela 1).
Dentre 973 indivíduos que estão inseridos formalmente no mercado de trabalho, 96 (5,2\%) trabalhavam em ambientes domiciliares, como domésticas/diaristas, e 37 (2\%) desenvolvem suas atividades laborativas em pet shop e clínicas veterinárias.

Quando foi estabelecida uma relação entre sexo e ocupação entre pessoas que estão fora do mercado de trabalho ou desenvolvem suas atividades laborativas no ambiente domiciliar, verificou-se uma maioria feminina, totalizandose 722 mulheres $(39,1 \%)$ e 192 homens $(10,4 \%)$ nessas condições.

Dos 1.848 casos, $65 \%$ possuíam gato e, dentre eles, $80,3 \%$ tiveram como fonte de infecção declarada o gato no ambiente domiciliar.

Ao detalhar a fonte de infecção encontrouse que $1.543(83,5 \%)$ tinham registros para essa variável. Ressaltamos que 305 prontuários não tinham a informação sobre a fonte de infecção, sendo então categorizados nas análises como missing.

No detalhamento da fonte de infecção, identificou-se que a maioria dos casos tinha registros de relatos de pacientes com trauma envolvendo animais no ambiente doméstico. Um mil duzentos e vinte seis casos relataram o trauma com gato como fonte de infecção $(66,34 \%)$; entre esses, 965 casos $(78,71 \%)$ estavam relacionados ao gato doméstico; 99 casos (8,07\%), ao gato de rua e 162 casos $(13,21 \%)$ não especificaram a procedência do animal. Cabe ressaltar que 307 casos (16,61\%) se referiram ao trauma em lidar com plantas ou terra. As situações classificadas como "outros" foram referentes à menção de picadas de insetos, aves ou acidentes com cachorros, equivalentes a 10 casos $(0,5 \%)$.

Na análise temporal (Tabela 1), quase não foi evidenciada mudança no perfil epidemiológico dos 1.848 casos, excetua-se a variável inserção do mercado de trabalho, na qual foi identificada uma inversão ao longo dos períodos. Entre 1997 e 2000, pacientes classificados como fora do mercado de trabalho representavam $37,1 \%$ dos pacientes, entre 2005 e 2007 chegaram a 43,7\%. Um aumento em torno de $20 \%$.

A distribuição da doença segundo os municípios de residência (Tabela 2) permitiu identificar o registro de casos em 36 dos 92 municípios (39\%) que compõem o Estado do Rio de Janeiro, com representatividade em todas as regiões fluminenses. Entretanto, 95,13\% dos casos eram de pacientes residentes em 11 municípios da Região Metropolitana I. Na Região Metropolitana II, apenas três municípios apresentaram registro de casos, mesmo assim com proporção bastante inferior à verificada na Região Metropolitana I (1,62\%). Na Região Serrana ocorreu 1,24\% dos 
Figura 1

Mapa de kernel dos casos de esporotricose, entre 1997-2007, segundo município de residência na Região Metropolitana do Estado do Rio de Janeiro, Brasil, com destaque das localidades de transmissão intensa.

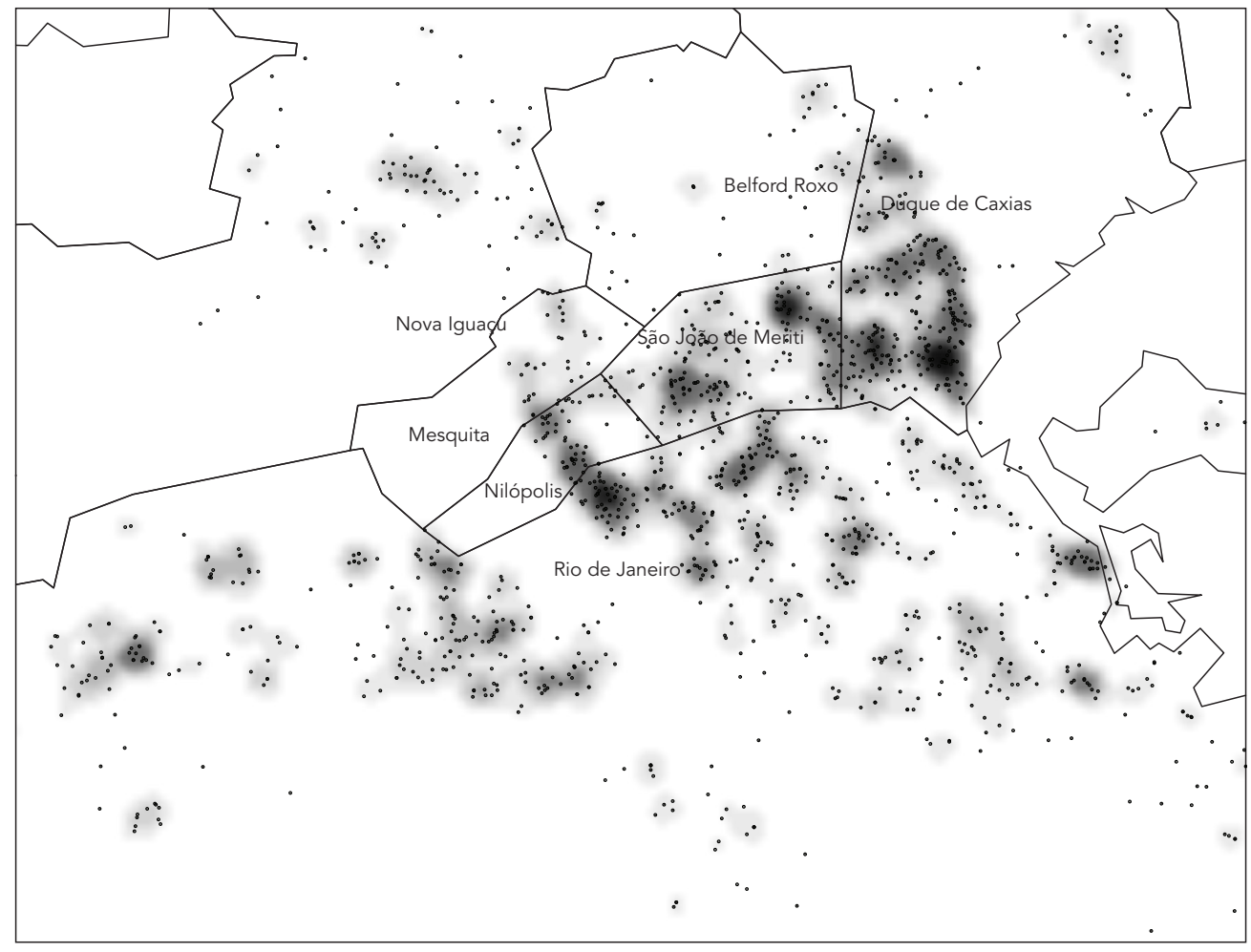

- Casos de esporotricose

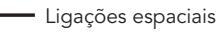

$\square$ Setor censitário

$\square$ Limites municipais

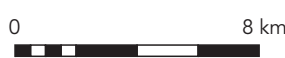

Kernel

$\square$ Baixa densidade

$\square$

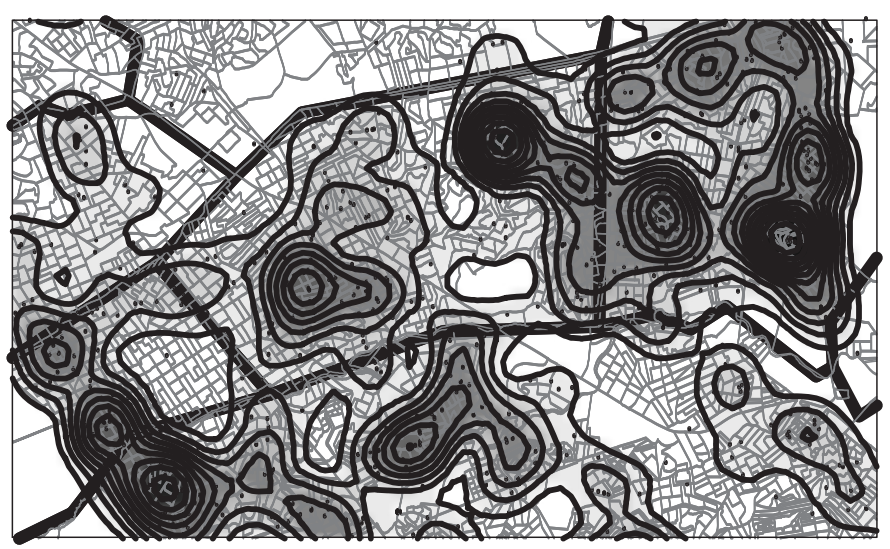

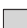

$\square$

$\square$ Média densidade

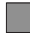

$\square$

$\square$

Alta densidade

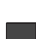

$\square$

\begin{tabular}{l}
$0.320 \mathrm{~m}$ \\
\hline
\end{tabular}
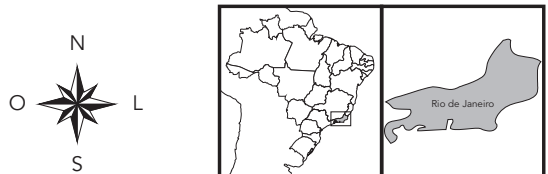
Distribuição por ano de notificação dos pacientes com esporotricose atendidos no Instituto de Pesquisa Clínica Evandro Chagas, Fundação Oswaldo Cruz, Rio de Janeiro, Brasil, 1997-2007.

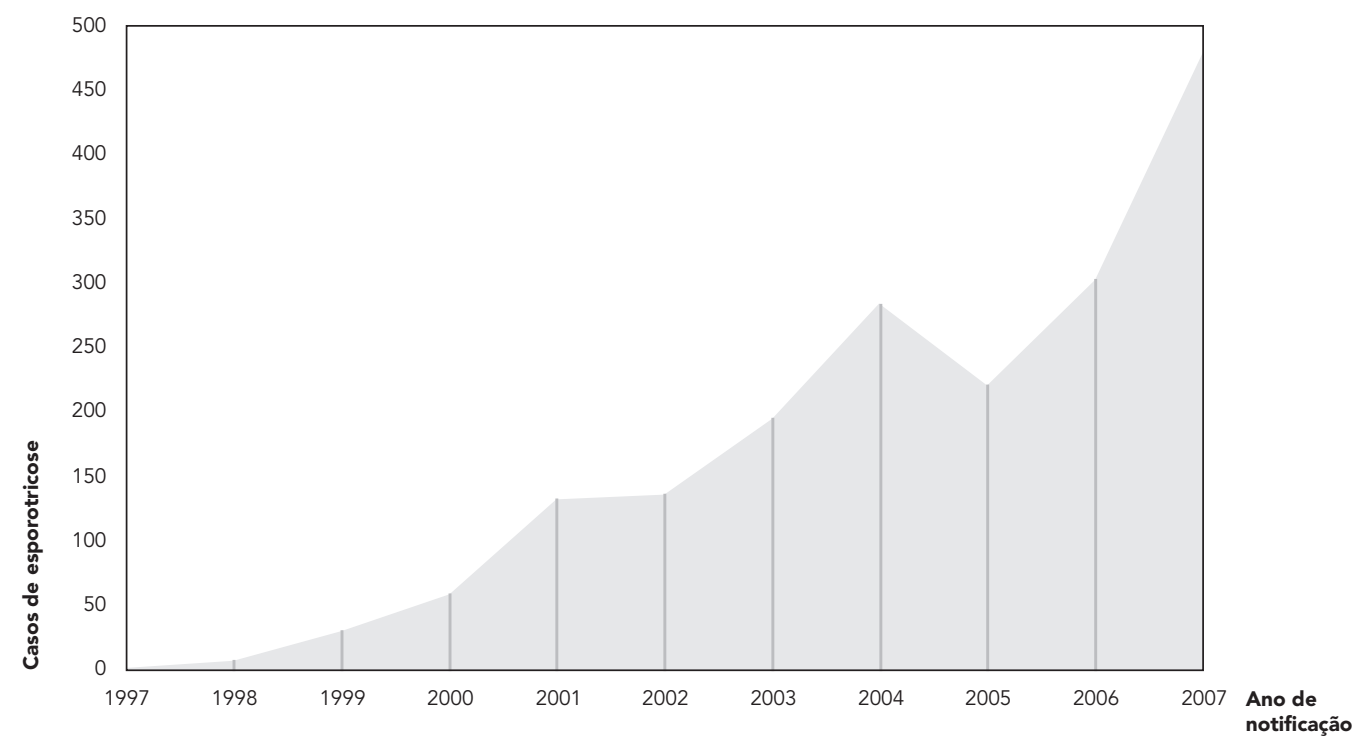

casos, na Costa Verde, 0,65\%, nas Baixadas Litorâneas $0,49 \%$, no Centro Sul Fluminense 0,32\%, no Médio-Paraíba 0,22\%, no Noroeste Fluminense, 0,16\% e no Norte Fluminense, também 0,16\%.

No período de 1997 a 2007, o Município do Rio Janeiro concentrou $51 \%$ do total de casos de esporotricose do estado. Em seguida, apareceram os municípios de Duque de Caxias (20,62\%), São João de Meriti (9,25\%), Nova Iguaçu (5,3\%), Nilópolis (3,95\%), Belford Roxo (3,08\%) e Mesquita (1,79\%). A distribuição dos casos da doença segundo três períodos (1997-2000, 2001-2004 e 2005-2007), permitiu verificar a elevação do número de casos na maioria dos municípios da Região Metropolitana I, salvo em Japeri.

Dos 1.747 casos residentes na Região Metropolitana I registrados no período do estudo, foi possivel georreferenciar 1.610 (92\%). Não se obteve $100 \%$ por conta da não disponibilização de malhas digitais de todos os municípios, como também em razão da incompletude da variável endereço de residência do paciente.

Com o georreferenciamento dos casos por setor censitário e com a construção do mapa de pontos e do mapa de kernel referente ao período total do estudo (1997-2007), foi possível identificar quatro áreas com alta densidade de casos de esporotricose, observando uma concentração em áreas limítrofes nos municípios do Rio de Janeiro: Duque de Caxias, Nilópolis e São João de Meriti (Figura 1).

Contudo, os mapas de kernel por períodos (1997-2000, 2001-2004 e 2005-2007) (Figura 3) permitiram observar uma variação na sua distribuição ao longo do tempo:

a) no primeiro período (1997-2000), a área de maior densidade de casos encontrava-se no município do Rio de Janeiro, mais especificamente no limite com o Município de Nilópolis. Outras duas áreas menos intensas foram observadas em São João de Meriti e Duque de Caxias (Figura 3); b) no segundo período (2001-2004), observaramse duas áreas de alta densidade e uma de média densidade no Município de Duque de Caxias. A área de alta densidade observada no período anterior, localizada no limite entre o Rio de Janeiro e Nilópolis, manteve-se presente, porém com intensidade média. Verificou-se uma área com média densidade no subúrbio da Leopoldina, mais especificamente no bairro de Ramos. Encontrouse uma difusão dos casos para a zona oeste da cidade do Rio de Janeiro, mais particularmente nos bairros de Realengo, Bangu e Campo Grande; c) no terceiro período, 2005-2007, a área com densidade alta esteve localizada em São João de Meriti. As áreas de alta densidade localizadas em 
Número e proporção de casos de esporotricose segundo algumas características sociodemográficas e fonte de infecção, segundo diferentes estratos de densidade e período. Instituto de Pesquisa Clínica Evandro Chagas, Fundação Oswaldo Cruz, Rio de Janeiro, Brasil, $1997-2007$.

\begin{tabular}{|c|c|c|c|c|c|c|c|c|}
\hline \multirow[t]{2}{*}{ Variáveis } & \multirow[t]{2}{*}{ n (\%) } & \multicolumn{4}{|c|}{ Estratos de densidade } & \multicolumn{3}{|c|}{ Período } \\
\hline & & Baixa & Média & Alta & $\begin{array}{l}\text { Fora do } \\
\text { kernel * }\end{array}$ & $1997-2000$ & 2001-2004 & 2005-2007 \\
\hline Total & $1.848(100,0)$ & $1.081(58,5)$ & $463(25,0)$ & $62(3,3)$ & $242(13,1)$ & $97(5,2)$ & $746(40,4)$ & $1.005(54,4)$ \\
\hline \multicolumn{9}{|l|}{ Sociodemográficas } \\
\hline \multicolumn{9}{|l|}{ Idade (anos) } \\
\hline$<10$ & $89(4,8)$ & $44(4,1)$ & $27(5,8)$ & $5(8,1)$ & $13(5,4)$ & $6(6,9)$ & $60(8,0)$ & $56(5,6)$ \\
\hline $11-20$ & $190(10,3)$ & $124(11,5)$ & $38(8,2)$ & $9(14,5)$ & $19(7,8)$ & $6(6,9)$ & $72(9,7)$ & $79(7,9)$ \\
\hline $21-60$ & $1.248(67,5)$ & $727(67,2)$ & $315(68,0)$ & $40(64,5)$ & $166(68,6)$ & $77(79,4)$ & $503(67,4)$ & $668(66,5)$ \\
\hline $61 \mathrm{e}+$ & $321(17,4)$ & $186(17,2)$ & $83(17,9)$ & $8(12,9)$ & $44(18,2)$ & $8(8,2)$ & $112(15,0)$ & $201(20,0)$ \\
\hline \multicolumn{9}{|l|}{ Sexo } \\
\hline Masculino & $612(33,1)$ & $365(33,8)$ & $140(30,2)$ & $17(27,4)$ & $90(37,2)$ & $30(30,9)$ & $251(33,6)$ & $331(32,9)$ \\
\hline Feminino & $1.237(66,9)$ & $716(66,2)$ & $323(69,8)$ & $45(72,6)$ & $152(62,8)$ & $67(69,1)$ & $496(66,5)$ & $673(67,0)$ \\
\hline \multicolumn{9}{|l|}{ Raça } \\
\hline Branca & $1.168(63,2)$ & $687(63,5)$ & $282(60,9)$ & $41(66,1)$ & $157(64,9)$ & $67(69,1)$ & $450(60,3)$ & $650(64,7)$ \\
\hline Negra & $175(9,5)$ & $112(10,4)$ & $37(7,9)$ & $5(8,1)$ & $21(8,7)$ & $3(3,1)$ & $84(11,3)$ & $88(8,8)$ \\
\hline Amarela & $7(0,4)$ & $5(0,5)$ & $1(0,2)$ & $1(1,6)$ & $0(0,0)$ & $0(0,0)$ & $6(0,8)$ & $1(0,1)$ \\
\hline Parda & $499(27,0)$ & $277(25,6)$ & $143(30,9)$ & $15(24,2)$ & $64(26,4)$ & $27(27,8)$ & $207(27,7)$ & $265(26,4)$ \\
\hline \multicolumn{9}{|l|}{$\begin{array}{l}\text { Escolaridade (anos de } \\
\text { estudo) }\end{array}$} \\
\hline 0 & $179(9,7)$ & $111(10,3)$ & $52(11,2)$ & $6(9,7)$ & $10(4,1)$ & $6(6,2)$ & $78(10,5)$ & $95(9,5)$ \\
\hline $1-3$ & $312(16,9)$ & $190(17,6)$ & $72(15,6)$ & $8(12,9)$ & $42(17,3)$ & $8(8,2)$ & $125(16,8)$ & $179(17,8)$ \\
\hline $4-7$ & $587(31,8)$ & $346(32,0)$ & $147(31,7)$ & $21(33,9)$ & $73(30,2)$ & $36(37,1)$ & $227(30,4)$ & $324(32,2)$ \\
\hline $8-11$ & $371(20,1)$ & $220(20,4)$ & $91(19,7)$ & $15(24,9)$ & $44(18,2)$ & $21(21,6)$ & $163(21,8)$ & $186(18,5)$ \\
\hline $12+$ & $301(16,3)$ & $161(14,9)$ & $72(15,6)$ & $9(14,5)$ & $60(24,8)$ & $17(17,5)$ & $113(15,1)$ & $171(17,0)$ \\
\hline NSA ** & $12(0,6)$ & $5(0,5)$ & $3(0,6)$ & $0(0,0)$ & $4(1,6)$ & $(0,0)$ & $6(0,8)$ & $6(0,6)$ \\
\hline Ignorado *** & $86(4,7)$ & $48(4,4)$ & $26(5,6)$ & $3(4,8)$ & $9(3,7)$ & $9(9,3)$ & $35(4,7)$ & $42(4,2)$ \\
\hline \multicolumn{9}{|l|}{$\begin{array}{l}\text { Situação no mercado de } \\
\text { trabalho }\end{array}$} \\
\hline Fora do mercado \# & $781(42,3)$ & $457(42,3)$ & $208(44,9)$ & $30(48,4)$ & $86(35,5)$ & $36(37,1)$ & $306(41,0)$ & $439(43,7)$ \\
\hline Formalmente empregado & $973(52,7)$ & $564(52,2)$ & $243(52,5)$ & $30(48,4)$ & $136(56,2)$ & $61(62,9)$ & $419(56,2)$ & $492(49,0)$ \\
\hline Ignorado *** & $94(5,1)$ & $60(5,5)$ & $12(2,6)$ & $2(3,2)$ & $20(8,3)$ & $0(0,0)$ & $22(2,9)$ & $73(7,3)$ \\
\hline \multicolumn{9}{|l|}{ Epidemiológicas } \\
\hline \multicolumn{9}{|l|}{ Fonte de infecção } \\
\hline Gato doméstico & $965(52,2)$ & $570(52,7)$ & $256(55,3)$ & $28(45,1)$ & $111(45,8)$ & $56(57,7)$ & $392(52,5)$ & $517(51,4)$ \\
\hline Gatos sem especificação & $162(8,8)$ & $98(9,0)$ & $37(8,0)$ & $3(4,8)$ & $24(9,9)$ & $6(6,2)$ & $40(5,4)$ & $116(11,5)$ \\
\hline Gato errante & $99(5,4)$ & $52(4,8)$ & $27(5,8)$ & $4(6,4)$ & $15(6,1)$ & $7(7,2)$ & $45(6,0)$ & $46(4,6)$ \\
\hline Solo ou plantas & $307(16,6)$ & $178(16,5)$ & $72(15,6)$ & $15(24,2)$ & $43(17,7)$ & $14(14,4)$ & $200(26,8)$ & $94(9,4)$ \\
\hline Outros & $10(0,5)$ & $7(0,6)$ & $3(0,6)$ & $0(0,0)$ & $0(0,0)$ & $1(1,0)$ & $3(0,4)$ & $6(0,6)$ \\
\hline Ignorado *** & $305(16,5)$ & $176(16,3)$ & $68(14,7)$ & $12(19,3)$ & $49(20,2)$ & $13(13,4)$ & $67(9,0)$ & $225(22,4)$ \\
\hline
\end{tabular}

NSA: não se aplica.

* Por não ter sido georreferenciado ou municípios fora da Região Metropolitana 1;

** Referente a crianças fora da idade escolar;

*** Não registrado no prontuário do paciente;

\# Desempregados, aposentados, pensionista, estudantes, donas de casa, crianças. 
Tabela 2

Distribuição dos casos de esporotricose por município de residência no Estado do Rio de Janeiro, Brasil. Instituto de Pesquisa Clínica Evandro Chagas, Fundação Oswaldo Cruz, 1997-2007.

\begin{tabular}{|c|c|c|c|c|c|c|}
\hline \multirow[t]{2}{*}{ Região/Município de residência } & \multirow[t]{2}{*}{$\mathbf{n}$} & \multirow[t]{2}{*}{$\%$ relativo } & \multirow[t]{2}{*}{$\%$ acumulado } & \multicolumn{3}{|c|}{ Período } \\
\hline & & & & $1997-2000$ & 2001-2004 & $2005-2007$ \\
\hline \multicolumn{7}{|l|}{ Metropolitana I } \\
\hline Rio de Janeiro & 934 & 50,54 & 50,54 & 35 & 377 & 522 \\
\hline Duque de Caxias & 381 & 20,62 & 71,16 & 27 & 181 & 173 \\
\hline São João de Meriti & 171 & 9,25 & 80,41 & 17 & 49 & 105 \\
\hline Nova Iguaçu & 98 & 5,30 & 85,71 & 0 & 36 & 62 \\
\hline Nilópolis & 73 & 3,95 & 89,66 & 4 & 38 & 31 \\
\hline Belford Roxo & 57 & 3,08 & 92,75 & 5 & 21 & 31 \\
\hline Mesquita & 33 & 1,79 & 94,53 & 1 & 11 & 21 \\
\hline Magé & 5 & 0,27 & 94,81 & 0 & 1 & 4 \\
\hline Seropédica & 3 & 0,16 & 94,97 & 0 & 1 & 2 \\
\hline Queimados & 2 & 0,11 & 95,08 & 0 & 0 & 2 \\
\hline Japeri & 1 & 0,05 & 95,13 & 0 & 1 & 0 \\
\hline \multicolumn{7}{|l|}{ Metropolitana II } \\
\hline São Gonçalo & 16 & 0,87 & 96,00 & 0 & 5 & 11 \\
\hline Niterói & 9 & 0,49 & 96,48 & 0 & 6 & 3 \\
\hline Itaboraí & 5 & 0,27 & 96,75 & 0 & 3 & 2 \\
\hline \multicolumn{7}{|l|}{ Baixada Litorânea } \\
\hline Saquarema & 4 & 0,22 & 96,97 & 0 & 0 & 4 \\
\hline Cabo Frio & 2 & 0,11 & 97,08 & 0 & 0 & 2 \\
\hline Araruama & 1 & 0,05 & 97,13 & 0 & 0 & 1 \\
\hline Arraial do Cabo & 1 & 0,05 & 97,19 & 0 & 0 & 1 \\
\hline Casimiro de Abreu & 1 & 0,05 & 97,24 & 0 & 0 & 1 \\
\hline \multicolumn{7}{|l|}{ Centro-sul Fluminense } \\
\hline Miguel Pereira & 2 & 0,11 & 97,35 & 1 & 1 & 0 \\
\hline Paty do Alferes & 2 & 0,11 & 97,46 & 1 & 1 & 0 \\
\hline Mendes & 1 & 0,05 & 97,51 & 0 & 1 & 0 \\
\hline Três Rios & 1 & 0,05 & 97,56 & 0 & 0 & 1 \\
\hline \multicolumn{7}{|l|}{ Costa Verde } \\
\hline Itaguaí & 10 & 0,54 & 98,11 & 0 & 2 & 8 \\
\hline Mangaratiba & 2 & 0,11 & 98,21 & 1 & 0 & 1 \\
\hline \multicolumn{7}{|l|}{ Médio Paraíba } \\
\hline Barra do Piraí & 2 & 0,11 & 98,32 & 0 & 2 & 0 \\
\hline Barra Mansa & 1 & 0,05 & 98,38 & 0 & 0 & 1 \\
\hline Volta Redonda & 1 & 0,05 & 98,43 & 0 & 0 & 1 \\
\hline \multicolumn{7}{|l|}{ Noroeste } \\
\hline São Fidelis & 3 & 0,16 & 98,59 & 0 & 0 & 3 \\
\hline \multicolumn{7}{|l|}{ Norte } \\
\hline Macaé & 3 & 0,16 & 98,76 & 0 & 0 & 3 \\
\hline \multicolumn{7}{|l|}{ Serrana } \\
\hline Teresópolis & 13 & 0,70 & 99,46 & 3 & 7 & 3 \\
\hline Petrópolis & 6 & 0,32 & 99,78 & 1 & 2 & 3 \\
\hline Bom Jardim & 1 & 0,05 & 99,84 & 1 & 0 & 0 \\
\hline Cantagalo & 1 & 0,05 & 99,89 & 0 & 0 & 1 \\
\hline Guapimirim & 1 & 0,05 & 99,95 & 0 & 0 & 1 \\
\hline Nova Friburgo & 1 & 0,05 & 100,00 & 0 & 0 & 1 \\
\hline Estado do Rio de Janeiro & 1.848 & 100,00 & & 97 & 746 & 1.005 \\
\hline
\end{tabular}


Figura 3

Mapa de kernel dos casos de esporotricose, nos períodos estudados, segundo município de residência na Região Metropolitana do Rio de Janeiro, Brasil.

3a) Período de 1997-2000

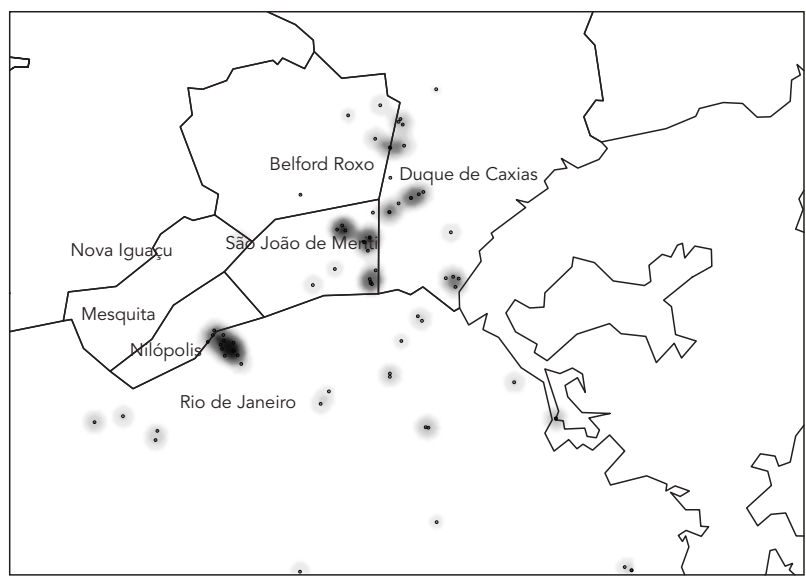

3b) Período de 2001-2004

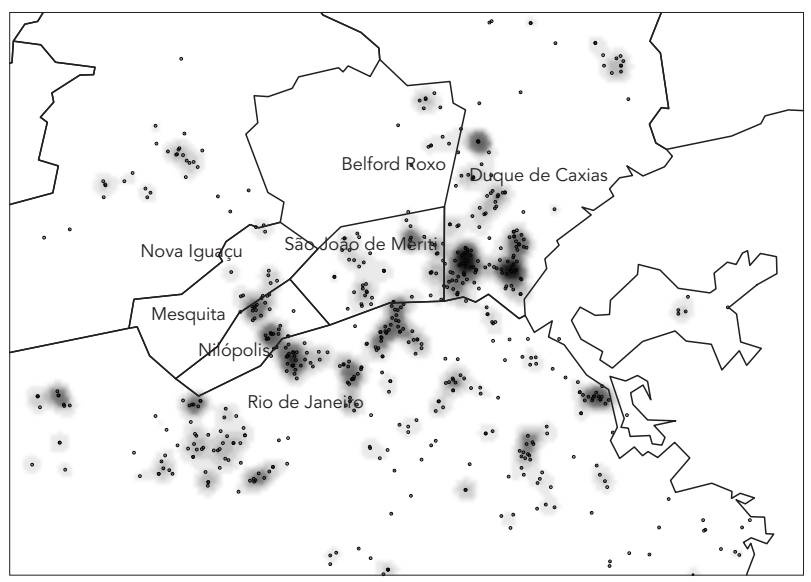

- Casos

Limites municipais

Kernel

Baixa densidade

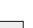

$\square$

(1)

3c) Período de 2005-2007
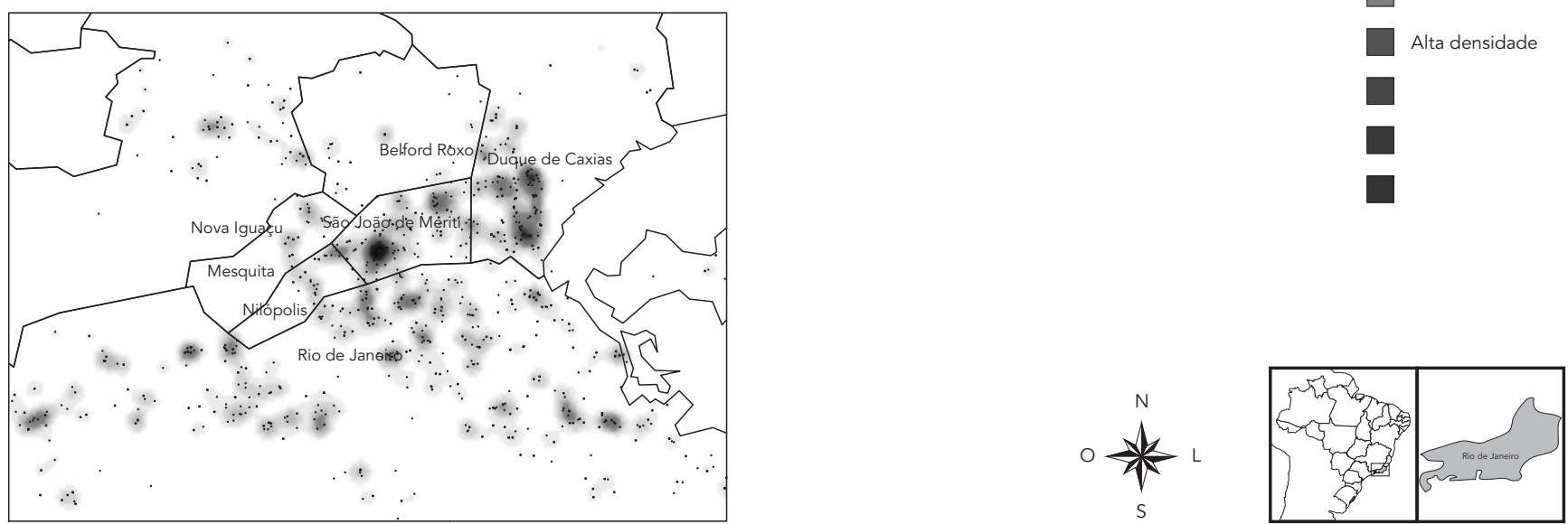
Duque de Caxias, observadas no período anterior, permaneceram ativas, mas com intensidade média (Figura 3). A área de alta densidade localizada no Rio de Janeiro, observada no primeiro e segundo períodos, manteve-se ativa, no entanto com baixa densidade. Verificou-se também no Município do Rio de Janeiro uma difusão na zona oeste, embora com baixa densidade, alcançando o bairro de Santa Cruz, bem como em alguns bairros do subúrbio da Leopoldina.

O georreferenciamento dos casos e análise de kernel permitiu delimitar diferentes estratos de densidade de casos, identificados pelos polígonos com distintas faixas de cores. Assim sendo, identificaram-se os casos de esporotricose dentro de cada estrato do kernel.

Visando verificar diferenças entre os estratos, foram realizadas análises que demonstraram que nos três níveis de densidade (alto/médio/baixo) a distribuição por sexo permaneceu na razão de duas mulheres para cada homem. Essas mulheres estão na faixa etária entre 21 e 60 anos, são predominantemente da raça branca e estão fora do mercado de trabalho (Tabela 1).

Para as localidades onde a densidade de esporotricose foi classificada como alta para o total do período (Figura 1), foram realizadas análises quanto às caracteristicas socioeconômicas, de infraestrutura e saneamento, utilizando dados do Censo Demográfico de $2000{ }^{24}$ por setores censitários. Tais localidades estão situadas nos municípios do Rio de Janeiro, São João de Meriti e Duque de Caxias. Oficialmente, elas não destoam das condições básicas de infraestrutura dos demais estratos nos municípios, excetuando os quesitos: esgoto ligado à rede geral e tipo de domicílio (Rio de Janeiro e Duque de Caxias).

Na localidade de alta densidade no Rio de Janeiro, somente 59,98\% dos domicílios estão ligados ao esgoto e $94,08 \%$ são domicílios do tipo casa. Em Duque de Caxias, oficialmente 96,61\% dos domicílios estão ligados à rede de esgotamento sanitário, e 95,47\% das moradias são do tipo casa.

Na capital Rio de Janeiro, a razão entre os responsáveis que recebiam até um salário mínimo e o total de responsáveis pelos domicílios foi de $12,39 \%$ e a densidade de pobres (responsáveis com um salário mínimo $/ \mathrm{km}^{2}$ ) foi de 627 . Valor bem diferente dos responsáveis para as localidades nos estratos médio e baixo do kernel do município (136).

Em Duque de Caxias, o percentual de responsáveis que recebiam até um salário mínimo foi de $20,89 \%$ no estrato alto. A densidade da população pobre situou-se em 1.529, bem diferente dos demais estratos do município, que registraram em torno de 7922 .

\section{Discussão}

A esporotricose humana no Rio de Janeiro vem sendo objeto de estudos há pelo menos uma década. Barros et al. ${ }^{3}$ descreveram 178 casos humanos atendidos pelo IPEC entre 1998 e 2001. Martinez 28 apresentou o seguimento dessa epidemia, com 572 casos entre 2002 e 2004. Em 2009, Freitas 9 deu continuidade ao estudo clínico, analisando 804 casos entre 2005 e 2008. Tais estudos focaram suas análises em variáveis clínicas da doença, já sendo considerada a maior epidemia por transmissão zoonótica 15.

O atual estudo buscou entender a distribuição geográfica da esporotricose no período de 1997-2007, extrapolando os limites geográficos do IPEC. A atual casuística contou 1848 casos de esporotricose. Entre 2008 e 2011, em período posterior à realização desta pesquisa, foram confirmados 2.340 novos casos de esporotricose humana, segundo dados do Serviço de Vigilância em Saúde do IPEC. A atualização dos dados indicou um possível incremento em sua incidência. Observou-se um aumento de $126,6 \%$ em relação à totalidade de casos dos 11 anos do estudo. Tal situação caracteriza esta epidemia como a maior epidemia mundial, ultrapassando os 4 mil casos.

Embora o nosso estudo não abranja outras unidades de atendimento de esporotricose públicas ou de consultórios privados, acredita-se que essa demanda nos demais locais não seja expressiva. Observa-se também que, ao longo dos anos, uma parte da nossa casuística era representada por este segmento, por meio do encaminhamento para a instituição, pois ela se tornou um centro de referência desses casos.

Ao resgatar a informação encontrada na literatura 13 sobre a distribuição por sexo, verifica-se um predomínio dos casos "em pacientes do sexo masculino e de idade mais avançada” 29 (p. 247) com mais de 50 anos. Neste estudo, identificamos um perfil diferente: a esporotricose vem se manifestando em mulheres, na idade adulta produtiva, porém fora do mercado de trabalho. Esse pode ser um indicativo de transmissão domiciliar da doença, fato que distingue esta casuística das já relatadas na literatura.

Durante anos, a esporotricose foi considerada uma doença ocupacional de manipuladores da terra 1,13 . Neste estudo, os trabalhadores que lidam com a terra representam apenas $0,48 \%$ dos registros, ou seja, 9 casos em 11 anos. Se a doença seguisse o curso de transmissão historicamente relatado 13 , tal seria a frequência esperada para o agravo no estado.

A esporotricose na região metropolitana do Estado do Rio de Janeiro ficou evidenciada, nesta última década, como uma doença urbana, não 
laborativa, de localidades nas quais, segundo informações oficiais, as condições de infraestrutura e saneamento são precárias 30 . A epidemia de esporotricose no estado se caracteriza pela transmissão zoonótica em ambiente domiciliar, tendo como transmissor o gato. O potencial de disseminação do fungo por intermédio do gato foi descrito por Schubach et al. ${ }^{31}$, que isolaram o fungo em $100 \%$ das lesões cutâneas felinas, $66,2 \%$ das cavidades nasais e $39,5 \%$ nas unhas dos gatos.

O contato dos indivíduos com os gatos no ambiente domiciliar talvez esteja aumentando nos últimos anos. Esse fato contribui para o aumento da possibilidade de infecção da população.

No Brasil, não existe um censo animal que permita conhecer por métodos diretos a quantidade existente de animais domésticos; fato que impede de determinar se a população felina vem crescendo ou diminuindo. Existem, isso sim, estimativas percentuais com base na população canina, que, por sua vez, são estimadas com base na população humana ${ }^{32}$, permitindo a programação das campanhas de vacinação.

Os relatórios das campanhas de vacinação antirrábica poderiam ser utilizados como formas indiretas de determinar o tamanho da população felina e seu incremento anual. O não acesso a esses relatórios foi um limitador neste estudo.

Identificou-se que a maior incidência de casos de esporotricose ocorre nos municípios da Região Metropolitana I, que é a de maior população e com uma ocupação urbana mais antiga 30 (Tabela 2).

A análise espacial da distribuição da doença, possibilitada por meio do georreferenciamento de casos, permitiu evidenciar uma faixa extensa com elevada densidade de casos no entorno da capital, a qual se denominou cinturão de esporotricose; ele extrapola os limites político-administrativos municipais existentes, indicando que essa é uma epidemia urbana que vem se espalhando silenciosamente neste início do século XXI.

A configuração deste cinturão ao longo dos limites entre a capital e os municípios vizinhos é desenhada por diversas áreas de densidade alta e média de casos de esporotricose. Chama a atenção que, apesar da baixa densidade, parece haver uma propagação da doença para bairros da zona oeste e para o subúrbio da Leopoldina. Contudo, a região que vai da área central à zona sul do Município do Rio de Janeiro não apresentou focos de densidade da doença neste período, provavelmente porque essas áreas possuem melhores condições socioeconômicas, de infraestrutura e habitação ${ }^{30}$, bem como características específicas de urbanização, como pavimentação completa e poucas áreas livres.
A esporotricose não se mostrou uma doença relacionada à escolaridade, mas sim influenciada pelos hábitos e estilos de vida. Está relacionada a populações pobres da cidade que têm uma determinada condição ambiental 30: pavimentação incompleta, casa com jardim, solo exposto e a presença de felino, fechando o ciclo gato-ambiente-homem.

Nossos resultados evidenciam que a esporotricose é uma doença epidêmica na região metropolitana do Rio de Janeiro, talvez em razão da presença do fungo no ambiente e das condições favoráveis à manutenção do seu ciclo de transmissão.

A esporotricose é uma doença negligenciada 33. Segundo a Organização Mundial da Saúde, doenças negligenciadas são doenças que não só prevalecem em condições de pobreza, mas também contribuem para a manutenção do quadro de desigualdade, pois representam forte entrave ao desenvolvimento dos países. Os investimentos em pesquisa e desenvolvimento não priorizam essa área, não despertam interesse das grandes empresas farmacêuticas multinacionais que não veem nessas doenças a oportunidade de obter compradores de seus produtos. O conhecimento produzido não se reverte em avanços terapêuticos, com novos fármacos e métodos diagnósticos 33,34. Já na década passada, Bustamante \& Campos 35 ressaltavam que a esporotricose é uma doença esquecida das agendas de pesquisa, visto que constitui um problema de saúde em somente algumas áreas geográficas e em desenvolvimento.

Com a difusão da doença em área urbana e a ausência de medidas adequadas de controle por parte do poder, aumenta a possibilidade de atingir grupos populacionais em situação de vulnerabilidade, levando, inclusive, a desenvolvimento de quadros mais graves da doença. Freitas et al. 9 relatam que em 2008 ocorreram dois óbitos em pacientes com a forma disseminada da doença e com outras morbidades associadas, como o HIV+. Esses óbitos são os primeiros relatados na literatura desta epidemia e pertencem à casuística do presente estudo.

Segundo Freitas et al. 9, 89,3\% dos doentes atendidos no IPEC entre 2005 e 2008, evoluíram para a cura, fazendo uso das drogas Itraconazol (100 ou $200 \mathrm{mg} / \mathrm{dia}$ ) ou Terbinafina $(250 \mathrm{mg} / \mathrm{dia})$, por um tempo médio de três meses.

O tratamento preconizado mais frequente é o Itraconazol (100mg/dia) 9, que custa em torno de $\mathrm{R} \$ 160,00$ (medicamento genérico cujo preço foi consultado em 17/Fev/2012. http://consul taremedios.com.br/medicamento/itraconazol/ RJ) por mês, somados a esse valor os exames complementares para diagnóstico e evolução da 
micose. Todos esses pontos devem ser considerados em estudo sobre avaliação do custo para diagnóstico e tratamento da esporotricose.

\section{Conclusões}

Nos últimos anos, no Estado do Rio de Janeiro, em especial na Região Metropolitana I, vem ocorrendo uma epidemia zoonótica de esporotricose que se encontra em franco crescimento e negligenciada pelo poder público. Por mais que se verifique um expressivo aumento no número de registro de casos, não existem medidas específicas que objetivem o seu controle.

A observação dos achados descritos evidenciou uma característica da esporotricose humana bastante distinta daquelas descritas na literatura científica sobre a doença no que se refere a sexo, faixa etária, ocupação e fonte de infecção, tendo se revelado uma doença prevalente em mulheres na idade adulta e que estão fora do mercado de trabalho.

Apesar de os estudos já publicados sobre a esporotricose no estado apontarem para um grave problema de saúde que perdura há mais de dez anos, poucos são os investimentos em pesquisas - que objetivem o desvendamento dos determinantes e condicionantes dessa epidemia - e, mais importante, em ações de controle da epidemia.
Sabemos que o controle da fonte de infecção é fundamental nesta situação.

Coloca-se como prioritária a investigação de fatores envolvidos na dinâmica de transmissão da doença, para auxiliar a decisão em relação às medidas de vigilância e controle necessárias para conter o seu crescimento. Deve-se ressaltar que a esporotricose é descrita na literatura como ocupacional rural e que sua ocorrência em área urbana no Estado do Rio de Janeiro nesta última década reforça a necessidade de estudos que identifiquem marcadores de evolução para esse deslocamento, bem como orientem a conduta para sua contenção.

A situação epidemiológica da esporotricose no estado e a particularidade do seu perfil epidemiológico e do seu padrão de transmissão colocam na agenda de pesquisa a necessidade de ampliação do conhecimento. O impacto da doença em nível individual e coletivo, na cidade e nos serviços de saúde do Rio de Janeiro, precisa ser descrito. Não há dúvidas quanto à importância de estudos interdisciplinares que possam integrar o conhecimento sobre o agravo, os quais relacionem as condições socioambientais e comportamentais que possam determinar a variação na transmissão do S. schenckii e como ela está relacionada à sua inserção socioespacial. Tais estudos devem ser realizados o mais breve possível.

\section{Resumo}

Na literatura científica, a esporotricose esteve associada por anos a profissionais que lidam com a terra, local onde o fungo causador habita. Recentemente, numa área urbana tem sido registrada a ocorrência relacionada à transmissão zoonótica. Este trabalho objetivou contribuir para o conhecimento sobre a esporotricose em área urbana, por intermédio da analise exploratória de sua distribuição socioespacial no Rio de Janeiro, Brasil, entre 1997 e 2007, identificando os espaços de transmissão mais intensos. Utilizando-se base de dados do Serviço de Vigilância em Saúde, Instituto de Pesquisa Clínica Evandro Chagas, Fundação Oswaldo Cruz, foram realizadas análises de frequên- cia da doença e sua distribuição espacial. No período estudado, foram registrados 1.848 casos de esporotricose, com predomínio em mulheres adultas fora do mercado de trabalho. A fonte de contaminação predominante foi ferimento causado pelo gato doméstico, o que contribuiu para a disseminação da esporotricose em área urbana. O georreferenciamento de 1.681 casos evidenciou um cinturão de transmissão ao longo da divisa entre a capital e os municípios da região metropolitana.

Esporotricose; Doenças do Gato; Vigilância Epidemiológica; Urbanização 


\section{Colaboradores}

M. B. T. Silva participou na concepção do projeto do artigo, levantamento de dados, análise dos dados, revisão crítica, redação e aprovação da versão final. M. M. M. Costa colaborou no levantamento de dados, análise estatística espacial, revisão crítica, redação e aprovação da versão final. C. C. S. Torres colaborou no levantamento de dados, análise estatística espacial, revisão crítica, redação e aprovação da versão final. M. C. G. Galhardo participou na concepção do projeto do artigo, análise dos dados, revisão crítica, redação e aprovação da versão final. A. C. F. Valle participou na concepção do projeto do artigo, redação e aprovação da versão final. M. A. F. M. Magalhães colaborou no levantamento de dados, análise estatística espacial, revisão crítica, redação e aprovação da versão final. P. C. Sabroza participou na concepção do projeto do artigo, revisão crítica e aprovação da versão final. R. M. Oliveira participou na concepção do projeto do artigo, análise dos dados, revisão crítica, redação e aprovação da versão final.

\section{Agradecimentos}

Ao Laboratório de Geoprocessamento do Instituto de Comunicação e Informação Científica e Tecnológica em Saúde, Fundação Oswaldo Cruz, onde todos os procedimentos de georreferenciamento e análise espacial foram realizados.

\section{Referências}

1. Schubach AO, Schubach TMP, Barros MBL, Wanke B. Esporotricose. In: Coura JR, organizador. Dinâmica das doenças infecciosas e parasitárias. Rio de Janeiro: Editora Guanabara Koogan; 2005. p. 1161-9.

2. Barros MBL, Schubach TMP, Galhardo MCG, Schubach AO, Monteiro PCF, Reis RS, et al. Sporotrichosis: an emergent zoonosis in Rio de Janeiro. Mem Inst Oswaldo Cruz 2001; 96:777-9.

3. Barros MBL, Schubach AO, Valle AC, Galhardo MCG, Conceição-Silva F, Schubach TMP, et al. Cattransmitted sporotrichosis epidemic in Rio de Janeiro, Brazil: description of a series of cases. Clin Infect Dis 2004; 38:529-35.

4. Schubach AO, Schubach TM, Barros MB. Epidemic cat-transmitted sporotrichosis. N Engl J Med 2005; 353:1185-6.

5. Barros MB, Costa DL, Schubach TM, Valle AC, Lorenzi NP, Teixeira JL, et al. Endemic of zoonotic sporotrichosis: profile of cases in children. Pediatr Infect Dis J 2008; 27:246-50.
6. Barros MBL, Schubach AO, Schubach TM, Wanke B, Lambert-Passos SR. An epidemic of sporotrichosis in Rio de Janeiro, Brazil: epidemiological aspects of a series of cases. Epidemiol Infect 2008; 136:1192-6.

7. Schubach AO, Barros MBL, Wanke B. Epidemic sporotrichosis. Curr Opin Infect Dis 2008; 21: 129-33.

8. Galhardo MC, Oliveira RM, Valle AC, Paes RA, Silvatavares PM, Monzon A, et al. Molecular epidemiology and antifungal susceptibility patterns of Sporothrix schenckii isolates from a cat transmitted epidemic of sporotrichosis in Rio de Janeiro, Brazil. Med Mycol 2008; 46:141-51.

9. Freitas DF, Valle AC, Almeida Paes R, Bastos FI, Galhardo MC. Zoonotic sporotrichosis in Rio de Janeiro, Brazil: a protracted epidemic yet to be curbed. Clin Infect Dis, 2010; 50:453.

10. Barros MBL, Schubach TP, Coll JO, Gremião ID, Wanke B, Schubach A. Esporotricose: a evolução e os desafios de uma epidemia. Rev Panam Salud Pública 2010; 27:455-60. 
11. Freitas DF, Siqueira Hoagland B, Valle AC, Fraga BB, Barros MB, Oliveira Schubach A, et al. Sporotrichosis in HIV-infected patients: report of 21 cases of endemic sporotrichosis in Rio de Janeiro, Brazil. Med Mycol 2012; 50:170-8.

12. Lopes JO, Alves SH, Mari CR, Brum LM, Westphalen JB, Altermann MJ, et al. Epidemiologia da esporotricose na região central do Rio Grande do Sul. Rev Soc Bras Med Trop 1999; 32:541-5.

13. Donadel KW, Reinoso YD, Oliveira JC, Azulay RD. Esporotricose: revisão. An Bras Dermatol 1993; 68:45-52.

14. Schubach TM, Valle ACF, Galhardo MCG, Fialho $\mathrm{PC}$, Reis R, Zancopé OR, et al. Isolation of Sporothrix schenckii from the nails of domestic cats (Felis catus). Med Mycol 2001; 39:147-9.

15. Schubach TMP, Schubach AO, Okamoto T, Barros MBL, Figueiredo FB, Cuzzi T, et al. Evaluation of an epidemic of sporotrichosis in cats: 347 cases (1998-2001). J Am Vet Med Assoc 2004; 224:1623-9.

16. Lyon GM, Zurita S, Casquero J, Holgado W, Guevara J, Brandt ME, et al. Population-based surveillance and a case-control study of risk factors for endemic lymphocutaneous sporotrichosis in Peru. Clin Infect Dis 2003; 36:34-9.

17. Xavier MO, Nobre MO, Sampaio Junior DP, Antunes TA, Nascente PS, Soria FBA, et al. Esporotricose felina com envolvimento humano na cidade de Pelotas, RS, Brasil. Ciênc Rural 2004; 34:1961-3.

18. Donadel KW, Oliveira JC, Mendonça IRSM, Azulay RD. Esporotricose na infância: relato de dois casos. An Bras Dermatol 1992; 67:121-5.

19. Simson FW. Sporotrichosis infection in mines of the Witwatersrand: a symposium. Johannesburg: Transvaal Chamber of Mines; 1947.

20. Bustamante B, Campos PEC. Endemic sporotrichosis. Current Opin Infect Dis 2001; 14:145-9.

21. Silva MBT. Distribuição socioespacial da esporotricose humana de pacientes atendidos no Instituto de Pesquisa Clínica Evandro Chagas, no período de 1997 a 2007, residentes no Estado do Rio de Janeiro [Dissertação de Mestrado]. Rio de Janeiro: Escola Nacional de Saúde Pública Sergio Arouca, Fundação Oswaldo Cruz; 2010.

22. Centro de Informações e Dados do Rio de Janeiro, Secretaria de Planejamento e Gestão. Estado do Rio de Janeiro, regiões de governo (descrição). http://www.observatoriobaixada.com.br/site/ images/files/cidade\%20e\%20meio\%20ambiente/ Estado\%20do\%20RJ\%20-\%20Regi\%C3\%B5es\%20 de\%20Governo,\%202009.pdf (acessado em 15/ Fev/2012).
23. Martins WJ, Albuquerque C, Fernandes VR. Plano diretor de regionalização. Rio de Janeiro: Governo do Estado do Rio de Janeiro; 2001.

24. Instituto Brasileiro de Geografia e Estatística. Base de informações por setor censitário da área urbana dos distritos-sede, censo demográfico 2000, resultados do universo. Região Sudeste - RJ/SP [DVD]. Rio de Janeiro: Instituto Brasileiro de Geografia e Estatística; s.d.

25. Miranda AC, Barcellos C, Moreira JC, Monken M. Território, ambiente e saúde. Rio de Janeiro: Editora Fiocruz; 2008.

26. Pedro AS, Souza-Santos RS, Sabroza PC, Oliveira RM. Condições particulares de produção e reprodução da dengue em nível local: estudo de Itaipu, Região Oceânica de Niterói, Rio de Janeiro, Brasil. Cad Saúde Pública 2009; 25:1937-46.

27. Souza-Santos R, Carvalho MS. Análise da distribuição espacial de larvas de Aedes aegypti na Ilha do Governador, Rio de Janeiro, Brasil. Cad Saúde Pública 2000 ; 16:31-42.

28. Martinez EZ. Perfil epidemiológico, clínico e terapêutico da Esporotricose no Instituto de Pesquisa Clínica Evandro Chagas - FIOCRUZ, Rio de Janeiro, no período de 2002 a 2004 [Dissertação de Mestrado]. Rio de Janeiro: Instituto Oswaldo Cruz, Fundação Oswaldo Cruz; 2006.

29. Lima LB, Pereira Júnior AC. Esporotricose: inquérito epidemiológico. Importância como doença profissional. An Bras Dermatol 1981; 56:243-8.

30. Marques EC. Estado e redes sociais: permeabilidade e coesão nas políticas urbanas no Rio de Janeiro. Rio de Janeiro: Revan; 2000.

31. Schubach TMP, Schubach AO, Reis RS, CuzziMaya T, Blanco TC, Monteiro DF, et al. Sporothrix schenckii isolated from domestic cats with and without sporotrichosis in Rio de Janeiro. Mycopathologia 2002; 153:83-6.

32. World Health Organization. Guidelines for dog population management. Geneva: World Health Organization; 1990.

33. Souza W. Doenças negligenciadas. Rio de Janeiro: Academia Brasileira de Ciências; 2010.

34. Departamento de Ciência e Tecnologia, Secretaria de Ciência, Tecnologia e Insumos Estratégicos, Ministério da Saúde. Doenças negligenciadas: estratégias do Ministério da Saúde. Rev Saúde Pública 2010; 44:200-2.

35. Bustamante B, Campos PE. Sporotrichosis: a forgotten disease in the drug research agenda. Expert Rev Anti Infect Ther 2004; 2:85-94.

Recebido em 22/Nov/2011

Versão final reapresentada em 15/Jun/2012

Aprovado em 13/Jul/2012 